\title{
Health-related quality of life, anxiety, and depressive symptoms in children with primary immunodeficiencies
}

This article was published in the following Dove Press journal:

Patient Preference and Adherence

17 March 2014

Number of times this article has been viewed

\author{
Nina B Kuburovic' \\ Srdjan Pasic ${ }^{2}$ \\ Gordana Susic ${ }^{3}$ \\ Dejan Stevanovic ${ }^{4}$ \\ Vladimir Kuburovic ${ }^{5}$ \\ Slavisa Zdravkovic ${ }^{6}$ \\ Mirjana Janicijevic Petrovic ${ }^{7}$ \\ Tatjana Pekmezovic ${ }^{8}$ \\ 'Department of Public Health, Mother \\ and Child Health Care Institute of \\ Serbia, ${ }^{2}$ Department of Immunology, \\ Pediatric Clinic, Faculty of Medicine, \\ University of Belgrade, ${ }^{3}$ Institute \\ for Rheumatology, Clinical Center \\ of Serbia, ${ }^{4}$ Clinic for Neurology \\ and Psychiatry for Children and \\ Adolescents, ${ }^{5}$ Pediatric Clinic, Mother \\ and Child Health Care Institute \\ of Serbia, ${ }^{6}$ Pediatric Day Hospital, \\ Mother and Child Health Care \\ Institute of Serbia, Belgrade, ${ }^{7}$ Faculty \\ of Medical Sciences, University of \\ Kragujevac, Kragujevac, ${ }^{8}$ Serbia \\ Institute of Epidemiology, Faculty \\ of Medicine, University of Belgrade, \\ Belgrade, Serbia
}

Correspondence: Nina B Kuburovic Department of Public Health, Mother and Child Health Care Institute of Serbia, 6-8 Radoja Dakića, Belgrade II070, Serbia Tel +381607187190

Fax +38 I I| 2697232

Email nina.kuburovic@gmail.com
Introduction: The aims of this study were to evaluate levels of health-related quality of life (HRQOL) and the presence of anxiety and depressive symptoms in children with primary immunodeficiency disease (PID) in Serbia.

Materials and methods: Self- and parent-rated data from 25 children with PID were available. As controls, data from 50 children with juvenile idiopathic arthritis (JIA) and 89 healthy children were included. The Pediatric Quality of Life Inventory was used for HRQOL assessments Anxiety symptoms were identified using the Screen for Child Anxiety-Related Emotional Disorders questionnaire, while depressive symptoms were identified using the Mood and Feeling Questionnaire.

Results: Children with PID had significantly lower Pediatric Quality of Life Inventory total scores compared to children with JIA and healthy children as child-rated $(P=0.02)$ and parentrated $(P<0.001)$. Specifically, they had significantly lowered emotional functioning compared to children with JIA, and social functioning compared to both children with JIA and healthy children. School functioning was significantly lower among children with PID (parent-rated only). By parent-rated responses, six (24\%) out of 25 children with PID had significant anxiety symptoms, while five $(20 \%)$ children had significant depressive symptoms, which was statistically higher than among children with JIA and healthy controls $(P=0.05)$.

Conclusion: HRQOL could be significantly compromised in children with PID, particularly across such psychosocial domains as emotional, social, and school. These children were also found to be at an increased risk for suffering significant anxiety and depressive symptoms.

Keywords: children, anxiety, depression, primary immunodeficiency disease, quality of life

\section{Introduction}

Primary immunodeficiency disease (PID) represents a heterogeneous group of rare inherited disorders of the immune system. ${ }^{1,2}$ According to the International Union of Immunological Societies, which is associated with the World Health Organization, all PID are classified based on the components of the immune system affected, such as antibody deficiencies, disorders of cellular immunity, or phagocytic and complement deficiencies. ${ }^{3,4}$ To date, over 200 different PIDs have been described and over 140 specific gene mutations have been identified. ${ }^{5}$ Considering these enormous numbers of different types and genetic causes of PID, there are great challenges in the diagnosis, treatment, and prognosis of pediatric cases. Firstly, the prevalence rates of PID in the general population have not been identified, and some authors have reported the prevalence rates from their national registries to be one per $10,000.5,6$ Considering the rarity of the disorder and the heterogeneity of the symptoms associated with it, 
mostly recurrent infections and propensity to malignancies, there is a low probability of correct diagnosis, even with diagnosis delay of over 5 years. ${ }^{5,6}$ Additionally, although onset in early years of life is most likely, PID can emerge at any age, which can be misleading for correct diagnosis and timely treatment. ${ }^{6}$ Finally, the prognosis of PID can vary significantly, from benign conditions, such as respiratory infection, to very complex conditions, such as malignancies with lethal outcome. ${ }^{6-8}$ Due to advances in PID treatment, primarily related to immunoglobulin therapy, morbidity and mortality rates have improved remarkably in recent years, and the majority of children (for brevity, we use "children" to include adolescents as well) with PID now survive into adulthood. ${ }^{9}$

In light of such challenges and taking into consideration the chronic nature of the disease, it is expected that PIDs affect the child's psychosocial development, such as self-perception, self-esteem, interpersonal relationships, and social activities, as observed in other pediatric conditions or in adults with PIDs. ${ }^{9-11}$ Therefore, it is evident that data about the outcomes of PID should be extended from simple clinical/ disease-parameter assessments to patient-reported outcome measures, providing information about how the child with PID and his/her family perceives the impact of disorders across different life domains. Health-related quality of life (HRQOL), a multidimensional construct covering physical, emotional, mental, social, and behavioral components of well-being and functioning, has been recognized as one of the most important patient-reported outcome measures for evaluating the outcome of a chronic pediatric condition. ${ }^{12,13}$ Considering that HRQOL data provide direct information about the impact of a disorder and its treatment, HRQOL assessments could be a part of multimodal assessment and treatment approach in children with PID as well. However, the evidence of to what extent PID affects HRQOL in children is limited, because only three studies that evaluated HRQOL in this population are available. ${ }^{14-16}$

In an American study from 2004, 28 children with different types of PIDs receiving intravenous immunoglobulin were assessed with the parent/proxy-reported 50-item Child Health Questionnaire Parent Form. ${ }^{14,17}$ In an Iranian study from 2006, 50 children with PID were evaluated with the self-reported Pediatric Quality of Life Inventory (PedsQL) questionnaire to assess HRQOL. ${ }^{15,18}$ Finally, in an Italian study from 2009, HRQOL was investigated in 25 children with X-linked agammaglobulinemia (XLA), a subgroup of PIDs. ${ }^{16}$ The authors used both child and parent reports of the PedsQL and its Rheumatology Module to assess HRQOL. ${ }^{16,19}$ Consistent findings across the studies are that the physical, psychological, and social domains of well-being and functioning among children with PIDs are significantly compromised. This observation could imply that including HRQOL data as a part of multimodal assessment and treatment approach in children with PID would be of great importance for planning treatment regimens.

However, before suggesting models for multimodal assessment and treatment that include HRQOL, more epidemiological studies are needed from different regions, considering the heterogeneous nature of the disorder, the small number of patients available for research, and some limitations of the previous studies. For example, although HRQOL in children with PID was evaluated compared to healthy children and to children with chronic conditions, not all previous studies included self/proxy-report data. ${ }^{14,15}$ Self-reporting is important, because data suggest that parents/proxies and children disagree when providing HRQOL data, and thus it is always necessary to include both in assessments. ${ }^{20}$ Additionally, children with chronic health conditions are at an increased risk for experiencing various psychological problems. ${ }^{21}$

Recent studies have emphasized that mental health plays an important role in HRQOL perceptions among both healthy children and children with chronic conditions. ${ }^{22-24}$ None of the past studies evaluated psychological problems in children with PID. Therefore, this study was designed to provide more data about HRQOL in children with PIDs. Levels of HRQOL in children with PIDs in Serbia, as well as levels of anxiety and depressive symptoms, were evaluated based on self- and parent ratings. It was assumed that children with PID would have lower levels of HRQOL and higher levels of anxiety and depressive symptoms than children with juvenile idiopathic arthritis (JIA) and healthy children, brought in as control groups in the study. Additionally, it was assumed that children with PIDs and higher levels of anxiety and depressive symptoms would have lower levels of HRQOL.

\section{Materials and methods \\ Participants}

Participants were pediatric patients with PIDs diagnosed and treated in the Mother and Child Health Care Institute of Serbia. All children, with at least one parent, were included between July 2011 and January 2012. The general studyinclusion criteria were, confirmed diagnosis of PID based on the criteria of the Pan-American Group for Immunodeficiency and the European Society for Immunodeficiencies, and 18 years of age as the upper limit. ${ }^{2}$ 
Children with JIA and healthy children, both with at least one parent, were the control groups in the study. Children with JIA were outpatients from the Institute of Rheumatology, Belgrade, Serbia, and all were recruited in the same period as children with PID. The general entry criteria for children with JIA were, confirmed JIA diagnosis established at least 6 months prior to the study, and 18 years of age as the upper limit. Healthy children were randomly chosen from one kindergarten and one elementary and one secondary school in the Belgrade region. The exclusion criteria for all children were, the presence of a progressive neuropsychiatric disorder, severe neurological impairments, intellectual disability, and inability to read and write Serbian (for 8-year-olds and parents).

Participation in the study was on a voluntary basis. After parents and children older than 14 years of age signed the consent, the questionnaires were completed. Children older than 8 years self-completed all questionnaires, as well as their parents. Children with PID and JIA and their parents completed the questionnaires separately during the regular clinical visits under the supervision of the principal author. Healthy children completed the questionnaires in school, their parents at home, and both returned the questionnaires to the psychologists responsible.

The study protocol was in keeping with the tenets of the Declaration of Helsinki and its later amendments. The study was approved by the Ethics Committee of the Medical School, University of Belgrade, Serbia.

\section{Questionnaires}

Pediatric Quality of Life Inventory generic core scales

The PedsQL was used for HRQOL assessments. The PedsQL is a self-rated and proxy-rated questionnaire, and both versions include 23 items in the following scales: physical functioning (eight items), emotional functioning (five items), social functioning (five items), and school functioning (five items). ${ }^{18}$ Within the scales, all items are in a 5 -point response-scale format $(0=$ never a problem, $1=$ almost never a problem, $2=$ sometimes a problem, $3=$ often a problem, $4=$ almost always a problem), and all are reverse scored and linearly transformed to a $0-100$ scale. Higher scores indicate better HRQOL. The total PedsQL score was computed as the sum of all items divided by the number of items answered. The validity and reliability of the PedsQL for healthy children and children with chronic diseases have been established. ${ }^{18,19}$ The PedsQL in Serbian has sound general psychometric properties. ${ }^{25}$ The Mapi
Research Trust granted permissions for use and provided the Serbian PedsQL.

\section{Screen for Child Anxiety-Related Emotional Disorders questionnaire}

Levels of anxiety symptoms were evaluated using the Screen for Child Anxiety-Related Emotional Disorders (SCARED) questionnaire. ${ }^{26}$ SCARED is a self-rated and proxy-rated 41-item questionnaire for assessment of the entire spectrum of childhood anxiety disorders. Items were scored on a 3 -point scale $(0=$ not true or hardly ever true, $1=$ somewhat true or sometimes true, $2=$ very true or often true), and the sum of all answered items gives the total SCARED score (possible range 0-82). The higher the SCARED scores, the higher the levels of anxiety symptoms. A total SCARED score $\geq 25$ was considered the cutoff score for an indication whether anxiety levels were clinically relevant. ${ }^{26}$ SCARED in Serbian had the appropriate psychometric properties. ${ }^{27}$

\section{Mood and Feeling Questionnaire}

Levels of depressive symptoms were evaluated using the Mood and Feeling Questionnaire (MFQ). ${ }^{28}$ The MFQ is a selfrated and proxy-rated questionnaire, with items reflecting the level of depression in children and adolescents. The self-rated version has 33 items, and the proxy-rated version 34 items. Each item was scored on a 3 -point scale $(0=$ not true, $1=$ sometimes, $2=$ true), and the sum of all answered items was the total MFQ score (possible range 0-66 for a child and 0-68 for a parent version). The higher the MFQ score, the higher the levels of depression. Total MFQ scores $\geq 21$ (MFQ parent form) and $\geq 27$ (MFQ child form) were considered cutoff scores for an indication of whether depression levels were clinically relevant. ${ }^{28,29}$ The MFQ in Serbian had the appropriate psychometric properties. ${ }^{27}$

\section{Data analysis}

The following independent variables were considered: years of age, sex, place of residence, mother's age and employment status, and family monthly income. Analysis of variance or $\chi^{2}$ was used to test for statistical differences in these variables among the groups.

Means and standard deviation were calculated for the PedsQL (physical, emotional, social and school functioning, and total), SCARED, and MFQ scores. Analysis of variance was used to test for statistical differences among the groups when considering the PedsQL scores, with demographic variables that statistically differed among the groups set 
as covariates. Children with significant anxiety symptoms (SCARED total score values $\geq 25$ ) and significant depressive symptoms (MFQ total score values $\geq 21 / 27$ ) were identified. Differences in PedsQL scores between children with and without significant anxiety and depressive symptoms were assessed using Student's $t$-test. Cohen's effect size corrected for sample size $(d)$ was computed and was interpreted as $0.2=$ small, $0.5=$ moderate, and $0.8=$ large effect ${ }^{30}$ All $P$-values $\leq 0.05$ were considered statistically significant.

\section{Results}

Data were available for 25 children with PID, 50 children with JIA, and 89 healthy children. Self-reports of 8 -year-olds and older were available for 19 children with PID, 35 children with JIA, and 77 healthy children. General characteristics of all children and parents are given in Table 1. Considering that there were statistically significant differences among the groups in sex, place of residence, mother's age, mother's employment, and family income, all subsequent analyses were statistically controlled for these variables. Table 2 shows the clinical characteristics of children with PID.

\section{HRQOL}

The PedsQL scores for the groups are shown in Table 3. Children with PID had significantly lower PedsQL total score compared to children with JIA and healthy children on childrated $(P=0.02)$ and parent-rated $(P<0.001)$ assessments. For specific scales, children with PID had significantly lower emotional functioning score compared to children with JIA, while the social functioning score was lower compared to both children with JIA and healthy children. The school functioning scores were significantly lower among children with PID (parent-rated only). There were no other significant differences.

\section{Anxiety and depressive symptoms}

Percentages of children with significant anxiety and depressive symptoms, as indicated by the cutoff values of SCARED and the MFQ, are given in Table 4. For self-reported assessments, five $(26.3 \%)$ and two (10.5\%) of 19 reported significant anxiety and depressive symptoms, respectively. For parent-rated assessments, six (24\%) of 25 children with PID reported significant anxiety symptoms, and five (20\%) had significant depressive symptoms. These rates were higher than those observed for the other two groups.

\section{HRQOL and levels of anxiety and depressive symptoms}

The PedsQL total score was significantly lower among children with scores above the SCARED cutoffs than among those whose scores were below for child- $(t=3.64$, $P=0.002, d=1.77)$ and parent-rated $(t=2.09, P=0.05, d=1.01)$ assessments. No statistically significant differences were found in the PedsQL and MFQ scores for either child- or parent-rated assessments (Table 5).

\section{Discussion}

In this study, we evaluated HRQOL and levels of anxiety and depressive symptoms in children with PID. Besides providing additional data to the limited literature about HRQOL in children with PID, this is the first study to report data about psychological problems in these children, which appeared to be high.

The results in our study showed that children with PID had significantly lower levels of overall HRQOL compared to healthy children, which is a finding consistent with previous reports. ${ }^{14-16}$ However, children with PID in our sample had significantly lower levels of overall HRQOL compared to children with JIA, contrary to the study of Zebracki et al, ${ }^{14}$

Table I General characteristics of all participants

\begin{tabular}{|c|c|c|c|c|}
\hline & $\begin{array}{l}\text { Children with } \\
\text { PID }(n=25)\end{array}$ & $\begin{array}{l}\text { Children with } \\
\text { JIA }(n=50)\end{array}$ & $\begin{array}{l}\text { Healthy } \\
\text { children }(n=89)\end{array}$ & $\begin{array}{l}\text { Test value }(d f) \\
(P \text {-value) }\end{array}$ \\
\hline Age, mean (SD), years & II.3 (4.2) & II $.4(4.3)$ & $12.4(4.2)$ & $F(2)=I .4(0.3)$ \\
\hline Sex, male/female, n (\%) & $21(84) / 4(16)$ & $24(48) / 26(52)$ & $53(59.6) / 36(40.4)$ & $\chi^{2}(2)=8.9(0.05)$ \\
\hline \multicolumn{5}{|l|}{ Place of residence } \\
\hline Rural/urban, n (\%) & $13(52) / / 2(48)$ & $13(26) / 37(74)$ & $7(7.9) / 82(92.1)$ & $\chi^{2}(2)=25.2(<0.001)$ \\
\hline Mother's age, mean (SD), years & $40.4(8.1)$ & $37.7(6.1)$ & $42.2(5.9)$ & $F(2)=7.9(<0.001)$ \\
\hline \multicolumn{5}{|l|}{ Mother's employment } \\
\hline Employed/unemployed, n (\%) & $10(40) / 15(60)$ & $25(50) / 25(50)$ & $82(92.1) / 7(7.9)$ & $\chi^{2}(2)=41.9(<0.001)$ \\
\hline Father's age, mean (SD), years & $45.0(9)$ & $42.2(7.1)$ & $45.2(7.6)$ & $F(2)=2.6(0.08)$ \\
\hline \multicolumn{5}{|l|}{ Father's employment } \\
\hline Employed/unemployed, n (\%) & $15(60) / 10(40)$ & $38(76) / 12(24)$ & $69(77.5) / 20(22.5)$ & $\chi^{2}(2)=3.3(0.2)$ \\
\hline Family monthly income, mean (SD), $€$ & $427.6(251.5)$ & $517.0(359.5)$ & $1032.6(531.9)$ & $F(2)=30.1(<0.001)$ \\
\hline
\end{tabular}

Abbreviations: PID, primary immunodeficiency disease; JIA, juvenile idiopathic arthritis; SD, standard deviation; df, degrees of freedom. 
Table 2 Basic clinical characteristics of PID children $(n=25)$

\begin{tabular}{ll}
\hline PID subtype & $\mathbf{n}(\%)$ \\
\hline X-linked agammaglobulinemia & $7(28 \%)$ \\
IgA deficiency & $3(12 \%)$ \\
Common-variable immunodeficiency & $5(20 \%)$ \\
Chronic granulomatous disease & $2(8 \%)$ \\
Ataxia-telangiectasia & $2(8 \%)$ \\
IgG deficiency & $4(16 \%)$ \\
Nijmegen breakage syndrome & $1(4 \%)$ \\
Di George syndrome & $1(4 \%)$ \\
PID duration, mean (SD), years & $5.8(4.5)$ \\
PID complications, $n$ (\%) & \\
Without complications & $4(16 \%)$ \\
Respiratory & $14(56 \%)$ \\
Respiratory and neurological & $6(24 \%)$ \\
Gastroenterological & $1(4 \%)$ \\
\hline
\end{tabular}

Abbreviations: PID, primary immunodeficiency disease; Ig, immunoglobulin; SD, standard deviation.

who reported that children with PID had similar overall HRQOL, and to the study of Soresina et al, ${ }^{16}$ who showed that HRQOL was better in a PID sample than in rheumatologic conditions. In view of this, HRQOL differences could be explained by the differences between the two diseases, probably dependent on disease severity. It could be assumed that our sample included more severely affected patients or that our sample was more heterogeneous than previous ones.

Nevertheless, particular HRQOL domains in children with PID were found to be differently affected than in children with JIA or healthy children. Physical functioning as an HRQOL domain was similar between children with PID and JIA, but poorer than in healthy children, confirming previous data. ${ }^{14}$ However, the children with XLA reported by the Italian authors perceived their physical health comparable to healthy children, but significantly better than in those with rheumatologic conditions. ${ }^{16}$ On the other hand, our children showed significantly lower levels of social functioning compared to the controls, but only lower levels of emotional functioning compared to children with JIA. In one previous study, children with PID had lower scores on emotional and social activities compared to healthy children and similar to children with rheumatologic conditions. ${ }^{16}$ Zebracki et al found that children with PID had significantly lower scores on school and social activities, limitations on parental time and family activities, and parental emotional distress domains of HRQOL compared to healthy children. ${ }^{14}$

Additionally, children with PID did not differ from controls in school functioning, as found in the child self-reports. This can be explained by the fact that children with PID had normal daily activities and attended school regularly, owing mostly to substitute-immunoglobulin therapy, which reduces the frequency of infections and prevents serious infections and complications, which in the final instance significantly affect HRQOL. Nevertheless, parents of children with PID reported significantly lowered functioning in the school domain compared to the JIA group and healthy children. This study also found illness-specific differences between children with PID and those with JIA, and similar to Zebracki et al's findings, showed that PedsQL may be sensitive to differences between two chronic health conditions, supporting HRQOL as a multidimensional concept. ${ }^{14}$

Considering these observations about particular HRQOL domains in line with previous findings, there are consistent data that pediatric PID is less likely to affect physical functioning compared to psychosocial functioning, which could be significantly impacted. Most likely, this could be explained by the characteristics of the disorder, which does not affect such physical functions as mobility or self-care. In pediatric conditions, HRQOL could be affected across all domains (eg, physical, emotional, social, etc), such as in

Table 3 PedsQL scores in the groups (means and standard deviations)

\begin{tabular}{|c|c|c|c|c|c|c|c|c|}
\hline \multirow[t]{2}{*}{ PedsQL ${ }^{18,19}$ score* } & \multicolumn{4}{|c|}{ Self-report } & \multicolumn{4}{|c|}{ Proxy report } \\
\hline & $\begin{array}{l}\text { Children } \\
\text { with PID, } \\
n=19\end{array}$ & $\begin{array}{l}\text { Children } \\
\text { with JIA, } \\
n=35\end{array}$ & $\begin{array}{l}\text { Healthy } \\
\text { children, } \\
\mathrm{n}=77\end{array}$ & $F$ (P-value) & $\begin{array}{l}\text { Children } \\
\text { with PID, } \\
n=25\end{array}$ & $\begin{array}{l}\text { Children } \\
\text { with JIA, } \\
n=50\end{array}$ & $\begin{array}{l}\text { Healthy } \\
\text { children, } \\
\mathrm{n}=89\end{array}$ & $F(P$-value $)$ \\
\hline Total & 74.7 (I5.6) & 83.5 (13.9) & $83.7(9.4)$ & $3.16(0.02)^{\mathrm{a}}$ & $66.9(17.5)$ & $81.8(13.2)$ & $83.9(12.3)$ & $8.47(<0.00 \mathrm{I})^{\mathrm{c}}$ \\
\hline Physical functioning & $77.5(16.9)$ & 77.5 (18.8) & $83.4(11.7)$ & $2.55(0.05)$ & $66.0(24.1)$ & $75.3(20.5)$ & $83.3(16.5)$ & $2.4 I(0.09)$ \\
\hline Emotional functioning & $69.2(20.4)$ & $82.4(20.8)$ & $75.3(16.5)$ & $2.23(0.07)$ & $63.0(21.7)$ & 75.5 (19.9) & $76.6(16.8)$ & $3.5 \mathrm{I}(0.005)^{\mathrm{d}}$ \\
\hline Social functioning & $79.7(24.5)$ & $89.1(15.5)$ & $93.6(8.1)$ & $4.05(<0.00 \mathrm{I})^{\mathrm{b}}$ & $77.4(20.9)$ & $92.1(11.3)$ & $92.4(13.2)$ & $4.77(<0.00 \mathrm{I})^{\mathrm{e}}$ \\
\hline School functioning & $72.4(23.4)$ & 84.7 (I4.9) & $82.3(16.0)$ & $2.22(0.07)$ & $61.0(27.3)$ & $84.2(15.3)$ & 83.7 (I5.8) & $9.34(<0.00 \mathrm{I})^{\mathrm{f}}$ \\
\hline
\end{tabular}

Notes: *Scores adjusted for sex, place of residence, mother's age, mother's employment, and family income; $P$ ID versus JIA $-P=0.006$, PID versus healthy - $P=0.02$, JIA versus healthy $-P=0.97$; ${ }^{\circ} P I D$ versus JIA $-P=0.03$, PID versus healthy $-P<0.00$ I, JIA versus healthy $-P=0.35$; ; $P$ ID versus JIA $-P<0.00$ I, $P$ ID versus healthy $P<0.00 \mathrm{I}$, JIA versus healthy $-P=0.98$; $\mathrm{d}$ IID versus JIA $-P=0.03$, $P I D$ versus healthy $-P=0.28$, JIA versus healthy $-P=0.98$; $\mathrm{e} I \mathrm{D}$ versus JIA $-P<0.00 \mathrm{I}$, $P$ ID versus healthy $P<0.00$ I, JIA versus healthy $-P=0.97$; ' $P$ ID versus JIA $-P<0.00$ I, $P$ ID versus healthy $-P<0.00$ I, JIA versus healthy $-P=0.62$.

Abbreviations: PedsQL, Pediatric Quality of Life Inventory; PID, primary immunodeficiency disease; JIA, juvenile idiopathic arthritis. 
Table 4 Distribution of SCARED and MFQ cutoff values* in the groups

\begin{tabular}{|c|c|c|c|}
\hline Version & PID (\%) & JIA (\%) & Healthy (\%) \\
\hline \multicolumn{4}{|c|}{ SCARED $^{26}$ score } \\
\hline Parent & $6(24)$ & $4(8)$ & $7(7.9)$ \\
\hline Child & $5(26.3)$ & $5(14.3)$ & $8(10.5)$ \\
\hline \multicolumn{4}{|c|}{$\mathrm{MFQ}^{28}$ score } \\
\hline Parent & $5(20)$ & $3(6)$ & $5(5.6)$ \\
\hline Child & $2(10.5)$ & $3(10)$ & $4(6)$ \\
\hline
\end{tabular}

Note: *Total SCARED score $\geq 25$, total MFQ score $\geq 2$ I (MFQ parent form), and $\geq 27$ (MFQ child form).

Abbreviations: SCARED, Screen for Child Anxiety-Related Emotional Disorders; MFQ, Mood and Feeling Questionnaire; PID, primary immunodeficiency disease; JIA, juvenile idiopathic arthritis.

epilepsy ${ }^{31}$ or cancer, ${ }^{32}$ more affected in physical domains, such as in cerebral palsy, ${ }^{33}$ or more affected in psychological domains, such as in emotional disorders. ${ }^{34}$

For the results of psychological symptoms in our sample, almost every fourth to fifth child with PID had clinically significant anxiety or depressive symptoms. Significant anxiety symptoms in children with PID were observed nearly two to three times more frequently than among children with JIA or healthy peers. Among children with PID, anxiety was reported more frequently than depressive symptoms. However, parents of children with PID reported depressive symptoms more frequently than the children themselves did. Additional findings were that children with significant anxiety symptoms, but not depressive, had significantly lower levels of HRQOL. Although these data were drawn from a small number of subjects based on a screening questionnaire,

Table 5 PedsQL total scores compared to SCARED and MFQ cutoff scores in the PID group

\begin{tabular}{|c|c|c|c|}
\hline & $\mathbf{n}$ & Mean (SD) & t-test, df (P-value), $d^{*}$ \\
\hline \multicolumn{4}{|l|}{ SCARED ${ }^{26}$ score } \\
\hline \multicolumn{4}{|l|}{ PedsQL ${ }^{18,19}$ total } \\
\hline \multicolumn{4}{|l|}{ Child report } \\
\hline Below cutoff & 14 & $80.70(12.28)$ & $3.64,17$ (0.002), 1.77 \\
\hline Above cutoff & 5 & $57.88(11.25)$ & \\
\hline \multicolumn{4}{|l|}{ Parent report } \\
\hline Below cutoff & 19 & $70.70(16.78)$ & $2.09,23$ (0.049), I.0I \\
\hline Above cutoff & 6 & $54.66(14.93)$ & \\
\hline \multicolumn{4}{|l|}{ MFQ $^{28}$ score } \\
\hline \multicolumn{4}{|l|}{ PedsQL total } \\
\hline \multicolumn{4}{|l|}{ Child report } \\
\hline Below cutoff & 17 & $75.82(16.17)$ & $0.91,17(0.37)$ \\
\hline Above cutoff & 2 & $65.16(0.22)$ & \\
\hline \multicolumn{4}{|l|}{ Parent report } \\
\hline Below cutoff & 20 & $69.24(16.65)$ & $1.39,23(0.18)$ \\
\hline Above cutoff & 5 & $57.28(19.46)$ & \\
\hline
\end{tabular}

Note: *Cohen's $d$ effect size. ${ }^{30}$

Abbreviations: PedsQL, Pediatric Quality of Life Inventory; PID, primary immunodeficiency disease; SCARED, Screen for Child Anxiety-Related Emotional Disorders; MFQ, Mood and Feeling Questionnaire; $d f$, degrees of freedom; $t$-test, Student's $t$-test. they could imply that children with PID are at an increased risk for developing internalizing symptoms, with anxiety disorders more likely than depression. Possible explanations for this observation could be that anxiety symptoms are a more common reaction to a severe chronic condition than depressive symptoms are; anxiety symptoms might be unwanted side effects of specific therapy, or the PID itself inherently has a greater risk for developing anxiety then depressive psychopathology. Additionally, anxiety symptoms might have dominated over depressive symptoms in this sample, due to parents' own emotional reactions about their child's medical condition, considering that some data indicate that parents of chronically ill children have more psychological problems. ${ }^{35}$ Several previous studies on other chronic conditions in children also reported that anxiety symptoms were more likely than depressive symptoms to affect HRQOL. ${ }^{22-24}$

Several potential clinical and treatment implications should be emphasized. Firstly, parents and children could under- or overreport some HRQOL domains; therefore, both should be considered in order to provide a more comprehensive picture of HRQOL in PIDs. Secondly, the most consistent finding is that children with PIDs have lower levels of overall HRQOL and emotional, social, and school functioning domains compared to healthy children. However, data suggest that children with PID could have lower, similar, or even better HRQOL than children with rheumatologic disorders, namely JIA. Therefore, data about HRQOL from children with JIA could be used as a reference for children with PID, rather than data from healthy peers, because of the nature of JIA, which can have many different forms, with different body parts affected and severity levels. Thirdly, emotional problems, in particular anxiety symptoms, might play an important role in PIDs and need to be ruled out in routine assessments, because they can significantly affect HRQOL. ${ }^{23}$

Several study limitations should be acknowledged. In general, PID represents one heterogeneous group of very rare inherent disorders of the immune system. As such, small numbers of patients and great heterogeneity among them could significantly affect HRQOL findings. Our sample of children with PID was also heterogeneous in relation to clinical characteristics, with several subtypes of PID, therefore warranting more studies that are similar in order to provide valid data for clinical practice. In our sample of patients affected by PID, there were more boys, 21 (84\%), which could be partially explained by the mode of inheritance of the disease. It is known that only male children inherit X chromosome-related diseases (seven children with XLA 
in our sample). Further, if the disease is heterogeneously inherited, either autosomal-dominant or -recessive or by $\mathrm{X}$-chromosome link as in chronic granulomatos disease, there will be more male children affected. ${ }^{6}$ Although it could be expected that both sexes are equally affected, in our sample, there were more male children. This could be explained by the fact that the disease in boys maybe manifests in a more severe clinical form, and consequently is more frequently diagnosed, so they are referred to a tertiary health institution, such as our institute.

Moreover, this was a cross-sectional study from a clinical population, and a follow-up was not organized to evaluate HRQOL changes over time. Finally, due to its small sample size, this study did not consider how specific factors, especially treatment options, influence HRQOL.

There are several potential directions for future investigation. The evaluation of HRQOL is an important outcome measure of child and family well-being and functioning. The assessment of emotional problems, especially anxiety symptoms, should be a routine procedure to be given for every child with PID, together with laboratory findings, lung-function values, and other results. Further, more studies with larger samples of children with clinically similar types of immune disorders are needed, followed by investigation of different sociodemographic and clinical factors, such as severity and duration of the disease, intravenous immunoglobulin-therapy duration, age at first hospitalization, age at first infection, age at establishing diagnosis, and occurrence of complications, in order to identify the risk factors for poor HRQOL. This would only be possible if such a study is organized on an international level to have more children available for more accurate data. Finally, it is also necessary to consider anxiety and depressive symptoms independently and as comorbid conditions, but also behavioral problems in correlation with physical function and health status, and overall functioning and well-being in PID.

In summary, HRQOL could be significantly compromised among children with PID, in particular across psychosocial domains, such as emotional, social, and school. Additionally, these children are at an increased risk for suffering significant anxiety or depressive symptoms. With more research data accumulated in the coming years, simple clinical assessments in children with PID, such as laboratory data or physical examinations, could be substituted with models of multimodal assessment and treatment approaches that also include data about HRQOL and mental health in order to provide better care to these children.

\section{Acknowledgments}

We are grateful to the patients and families for their generous cooperation. The authors specially thank Mrs Zdravka Andric, school counselor, and the staff of the Radoje Domanovic primary school for the great support during the study. The authors would like to thank Mrs Branka Krsmanovic for her excellent assistance in preparing the manuscript. Tatjana Pekmezovic was supported by a grant from the Ministry of Science and Education of the Republic of Serbia (grant 175087). We are thankful to Luisacristina Carrillo-Marciano for English editing.

\section{Disclosure}

The authors report no conflicts of interest in this work.

\section{References}

1. Chapel H, Geha RS, Rosen F. Primary immunodeficiency disease: an update. Clin Exp Immunol. 2003;32:9-15.

2. Conly ME, Notarangelo LD, Etzioni A. Diagnostic criteria for primary immunodeficiencies. Representing PAGID (Pan-American Group for Immunodeficiency) and ESID (European Society for Immunodeficiencies). Clin Immunol. 1999;93:190-197.

3. Geha RS, Notarangelo LD, Casanova JL, et al. Primary immunodeficiency diseases: an update from the International Union of Immunological Societies Primary Immunodeficiency Diseases Classification Committee Meeting in Budapest, 2005. JAllergy Clin Immunol. 2007;120:776-794.

4. Chapel H. Classification of primary immunodeficiency disease by the International Union of Immunological Societies (IUIS) Expert Committee on Primary Immunodeficiency 2011. Clin Exp Immunol. 2012;168: 58-59.

5. Gathmann B, Grimbacher B, Beauté J, et al. European internet-based patient and research database for primary immunodeficiencies: results 2006-08. Clin Exp Immunol. 2009;157:3-11.

6. Lindegren ML, Kobrynski L, Rasmussen SA, et al. Applying public health strategies to primary immunodeficiency diseases: a potential approach to genetic disorders. MMWR Recomm Rep. 2004;53: $1-29$.

7. Sandoval C, Swift M. Hodgkin disease in ataxia-telangiectasia patients with poor outcomes. Med Pediatr Oncol. 2003;40:162-166.

8. Chrzanowska KH, Gregorek H, Dembowska-Bagińska B, Kalina MA, Digweed M. Nijmegen breakage syndrome (NBS). Orphanet J Rare Dis. 2012;7:13-25.

9. Sigstad HM, Stray-Pedersen A, Frøland SS. Coping, quality of life, and hope in adults with primary antibody deficiencies. Health Qual Life Outcomes. 2005;3:31.

10. Varni JW, Limbers CA, Burwinkle TM. Impaired health-related quality of life in children and adolescents with chronic conditions: a comparative analysis of 10 disease clusters and 33 disease categories/ severities utilizing the PedsQL 4.0 Generic Core Scales. Health Qual Life Outcomes. 2007;5:43.

11. Ingerski LM, Modi AC, Hood KK, et al. Health-related quality of life across pediatric chronic conditions. J Pediatr. 2010;156:639-644.

12. Ravens-Sieberer U, Erhart M, Wille N, Wetzel R, Nickel J, Bullinger M. Generic health-related quality of life assessment in children and adolescents: methodological considerations. Pharmacoeconomics. 2006;24:1199-1220.

13. Lovell DJ, Passo MH, Beukelman T, et al. Measuring process of arthritis care: a proposed set of quality measures for the process of care in juvenile idiopathic arthritis. Arthritis Care Res (Hoboken). 2011;63: $10-16$. 
14. Zebracki K, Palermo TM, Hostoffer R, Duff K, Drotar D. Healthrelated quality of life of children with primary immunodeficiency disease: comparison study. Ann Allergy Asthma Immunol. 2004;93: 557-562.

15. Mozaffari H, Pourpak Z, Pourseyed S, et al. Health-related quality of life in primary immune deficient patients. Iran J Allergy Asthma Immunol. $2006 ; 5: 23-27$

16. Soresina A, Nacinovich R, Bomba M, et al. The quality of life of children and adolescents with X-linked agammaglobulinemia. J Clin Immunol. 2009;29:501-507.

17. Landgraf JM, Abetz L, Ware JE. The Child Health Questionnaire: A User's Manual. Boston: Health Institute, New England Medical Center; 1996.

18. Varni JW, Seid M, Kurtin PS. PedsQL 4.0: reliability and validity of the Pediatric Quality of Life Inventory version 4.0 generic core scales in healthy and patient populations. Med Care. 2001;39:800-812.

19. Varni JW, Seid M, Smith Knight T, Burwinkle T, Brown J, Szer IS. The PedsQL in pediatric rheumatology. Reliability, validity, and responsiveness of the Pediatric Quality of Life Inventory Generic Core Scales and Rheumatology Module. Arthritis Rheum. 2002;46:714-725.

20. Upton P, Lawford J, Eiser C. Parent-child agreement across child healthrelated quality of life instruments: a review of the literature. Qual Life Res. 2008;17:895-913.

21. Lavigne J, Faier-Routman J. Psychological adjustment to pediatric physical disorders: a meta-analytic review. J Pediatr Psychol. 1992;17: 133-157.

22. Stevanovic D, Susic G. Health-related quality of life and emotional problems in juvenile idiopathic arthritis. Qual Life Res. 2012;22: 607-612.

23. Stevanovic D. Impact of emotional and behavioral symptoms on quality of life in children and adolescents. Qual Life Res. 2012;22: 333-337.

24. Stevanovic D, Jancic J, Lakic A. The impact of depression and anxiety disorders symptoms on the health-related quality of life of children and adolescents with epilepsy. Epilepsia. 2011;52:e75-e78.
25. Stevanović D, Lakić A, Damnjanović M. Some psychometric properties of the Pediatric Quality of Life Inventory ${ }^{\mathrm{TM}}$ Version 4.0 Generic Core Scales (PedsQL ${ }^{\mathrm{TM}}$ ) in the general Serbian population. Qual Life Res. 2011;20:945-949.

26. Birmaher B, Brent DA, Chiappetta L, Bridge J, Monga S, Baugher M. Psychometric properties of the Screen for Child Anxiety Related Emotional Disorders (SCARED): a replication study. Am Acad Child Adolesc Psychiatry. 1999;38:1230-1236.

27. Stevanovic D. Childhood depression and anxiety disorders in Serbia: a psychometric study of four screening questionnaires. Epidemiol Psychiatr Sci. 2011;21:111-116.

28. Wood A, Kroll L, Moore A, Harrington R. Properties of the mood and feelings questionnaire in adolescent psychiatric outpatients: a research note. J Child Psychol Psychiatr. 1995;36:327-334.

29. Kent L, Vostanis P, Feehan C. Detection of major and minor depression in children and adolescents: evaluation of the Mood and Feelings Questionnaire. J Child Psychol Psychiatry. 1997;38:565-573.

30. Cohen J. Statistical Power Analysis for the Behavioral Sciences. 2nd ed. Hillsdale (NJ): Lawrence Erlbaum Associates; 1988.

31. Stevanovic D, Tadic I, Novakovic T. Health-related quality of life in children and adolescents with epilepsy: a systematic review. In: Gadže ZP, editor. Epilepsy in Children - Clinical and Social Aspects. Rijeka, Croatia: InTech; 2011.

32. Sato I, Higuchi A, Yanagisawa T, et al. Cancer-specific health-related quality of life in children with brain tumors. Qual Life Res. Epub October 7, 2013.

33. Tantilipikorn P, Watter P, Prasertsukdee S. Comparison between utility of the Thai Pediatric Quality of Life Inventory 4.0 Generic Core Scales and 3.0 Cerebral Palsy Module. Int J Rehabil Res. 2013;36:21-29.

34. Stevanovic D, Tadic I, Knez R. Are antidepressants effective in quality of life improvement among children and adolescents? A systematic review. CNS Spectr. Epub September 13, 2013.

35. Moreira H, Carona C, Silva N, Frontini R, Bullinger M, Canavarro MC. Psychological and quality of life outcomes in pediatric populations: a parent-child perspective. J Pediatr. 2013;163:1471-1478.
Patient Preference and Adherence

\section{Publish your work in this journal}

Patient Preference and Adherence is an international, peer-reviewed, open access journal focusing on the growing importance of patient preference and adherence throughout the therapeutic continuum. Patient satisfaction, acceptability, quality of life, compliance, persistence and their role in developing new therapeutic modalities and compounds to

\section{Dovepress}

optimize clinical outcomes for existing disease states are major areas of interest. This journal has been accepted for indexing on PubMed Central. The manuscript management system is completely online and includes a very quick and fair peer-review system. Visit http://www.dovepress.com/ testimonials.php to read real quotes from published authors. 